\title{
Equalização de Canal Usando um Algoritmo LMS Largamente Linear Multi Split
}

\author{
Francisco J. A. de Aquino, Carlos A. F. da Rocha e Leonardo S. Resende
}

\begin{abstract}
Resumo - Neste artigo, apresentamos uma implementação multi-split de um equalizador largamente linear com baixa complexidade computacional. $O$ esquema considerado apresenta uma taxa de convergência que pode ser melhor que aquela obtida por um algoritmo LMS largamente linear normalizado. Os resultados de simulação comprovam o desempenho do algoritmo LMS largamente linear multi-split (LMS-LL-MS) para a equalização de um canal complexo quando a constelação empregada é real, como, por exemplo, uma constelação 4-PAM.
\end{abstract}

Palavras-Chave - Algoritmo LMS, filtragem adaptativa, processamento largamente linear, transformada multi-split.

Abstract - In this paper we present an implementation multi-split of a widely linear equalizer with low computational complexity. The considered scheme presents a convergence rate that can be better that one with normalized algorithm WL-LMS. Simulation results assess the performance of the multi-split WL-LMS algorithm for complex channel equalization when the symbol constellation is real as, for example, a 4-PAM constellation.

Keywords - LMS algorithm, adaptive filtering, widely linear processing, multi-split transform.

\section{INTRODUÇÃO}

Nos últimos anos, vários pesquisadores têm proposto o uso do processamento largamente linear como uma forma de aumentar o desempenho de equalizadores [1 - 5], quando o sinal recebido é impróprio [6]. Um exemplo de sinal impróprio ocorre quando um sinal real (sinal pertencente a uma constelação PAM, por exemplo) é transmitido por um canal complexo. Outros exemplos importantes são o caso de uma transmissão OQAM (offset quadrature amplitude modulation), GSM (Global System for Mobile Communication), e sistemas que transmitem em CPM (Continuous-Phase Modulation) binário com índice de modulação $h=1 / 2$, como mostrado em [3].

Neste artigo usamos a filtragem multi-split (MS) com parâmetros complexos, como apresentado em [7], aplicado ao algoritmo LMS largamente linear (LMS-LL). O algoritmo LMS-LL-MS (LMS-LL multi-split) pode apresentar uma taxa de convergência maior que o LMS-LL

Este trabalho foi parcialmente financiado pelo Centro Federal de Educação Tecnológica do Ceará (CEFET-Ce), Universidade Federal de Santa Catarina (UFSC) e Conselho Nacional de Desenvolvimento Científico e Tecnológico (CNPq) processo número 484391/2006-2.

F. J. A. de Aquino é professor no CEFET-Ce e aluno de doutorado na UFSC no Grupo de Pesquisa em Comunicação (GPqCom), e-mail: fcoalvesaq@cefet-ce.br. C. A. Rocha e L. S. Resende são professores no Departamento de Engenharia Elétrica da UFSC, laboratório GPqCom. Emails: \{aurelio, leonardo\}@eel.ufsc.br. normalizado [8] a um custo computacional pequeno. Vale a pena ressaltar que a aplicação da transformada mult-split ao processamento largamente linear ainda não foi considerada na literatura.

Este artigo é organizado como segue. A Seção II apresenta o algoritmo LMS multi-split. A Seção III apresenta de forma resumida os conceitos fundamentais do processamento largamente linear. A Seção IV apresenta o algoritmo LMS-LL aplicado ao problema de equalização de canais de comunicação. Na Seção $\mathrm{V}$ apresentamos o algoritmo LMS-LL-MS e os resultados de simulação. Finalmente, na Seção VI, concluímos este artigo.

\section{Algoritmo LMS MUlti-Split}

O algoritmo LMS é um importante membro da família de algoritmos de gradiente estocástico. Uma característica importante do algoritmo LMS é a sua simplicidade. Além disso, ele não requer medidas de funções de correlação, nem envolve a inversão de matrizes. Infelizmente, seu desempenho em termos de capacidade de rastreamento e taxa de convergência depende do espalhamento dos autovalores da matriz de correlação do sinal de entrada [9] e da escolha do passo de adaptação.

Teoricamente, usando uma transformação ortogonal como, por exemplo, a transformação de Karhunen-Loève (KLT), é possível obter uma diagonalização da matriz de autocorrelação do sinal e reduzir a relação dos autovalores e assim melhorar a taxa de convergência do algoritmo LMS. Porém, a transformação KLT apresenta uma complexidade computacional alta. Por outro lado, a operação de multi-split pode melhorar o desempenho do algoritmo de LMS com um baixo custo computacional [7].

A Fig. 1 mostra o esquema clássico de filtragem adaptativa usando um filtro transversal. Neste esquema, $n$ é o tempo discreto e um único sinal de erro é usado para adaptar todos os coeficientes do equalizador na mesma iteração. Usando a técnica multi-split, cada coeficiente do filtro é ajustado de forma independente. A operação multisplit, quando o número de coeficientes do equalizador é $N=$ $2^{M}, M \geq 1$, pode ser representada de uma forma compacta como mostrado na Fig. 2.

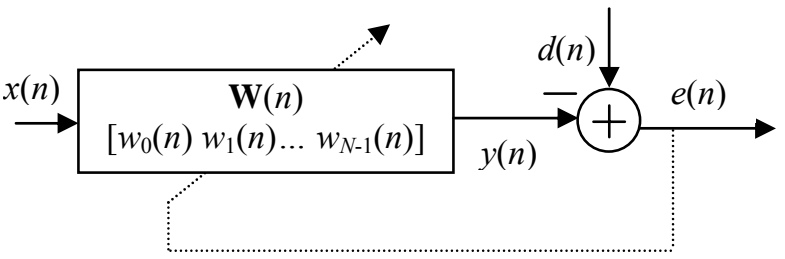

Fig. 1. Filtro transversal adaptativo clássico treinado. 


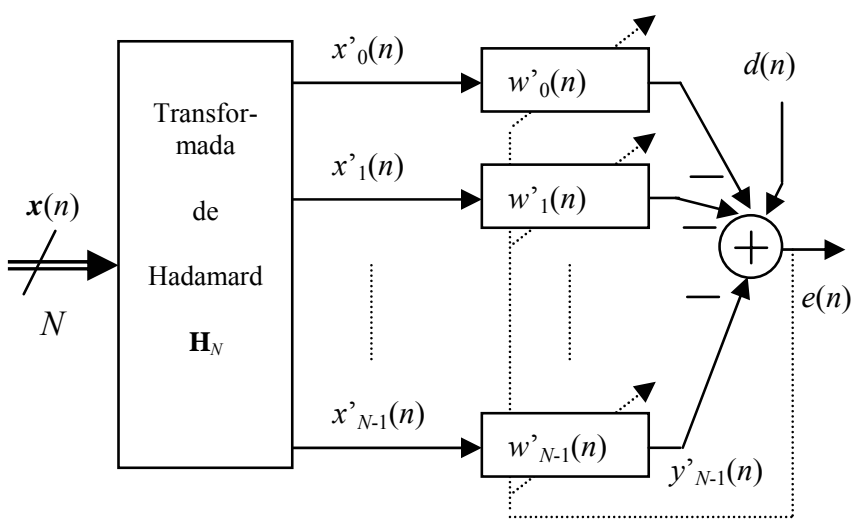

Fig. 2. Transformada de Hadamard da entrada $\boldsymbol{x}(n)$.

Neste caso, o procedimento multi-split é equivalente à aplicação da matriz de transformada discreta de WalshHadamard, o que é bem conveniente para a implementação em circuitos integrados (DSPs, FPGAs).

A matriz de Hadamard $\mathbf{H}_{N}$ de ordem $N$ pode ser formada a partir da matriz $\mathbf{H}_{N / 2}$ como segue:

$$
\mathbf{H}_{N}=\left[\begin{array}{cc}
\mathbf{H}_{N / 2} & \mathbf{H}_{N / 2} \\
\mathbf{H}_{N / 2} & -\mathbf{H}_{N / 2}
\end{array}\right],
$$

começando com $\mathbf{H}_{1}=$ [1]. Este procedimento pode gerar todas as matrizes de Hadamard que são potência de 2 . Podemos verificar facilmente que $\mathbf{H}_{N} \mathbf{H}_{N}=N \mathbf{I}$, onde I é uma matriz identidade $N \times N$.

Portanto, o procedimento multi-split pode ser visto como uma transformação linear dos dados de entrada dada por:

$$
\boldsymbol{x}^{\prime}(n)=\mathbf{H}_{N} \boldsymbol{x}(n),
$$

onde $\boldsymbol{x}(n)=[x(0) x(1) \ldots x(n-N+1)]^{T}$ é um vetor contendo os dados de entrada.

O procedimento multi-split aumenta a diagonalização da matriz de autocorrelação do sinal de entrada [10], e o algoritmo LMS pode ser usado para adaptar individualmente os parâmetros do equalizador, como mostra a Fig. 2. Estes fatos levam a um melhor desempenho do algoritmo LMS multi-split em relação ao LMS convencional. Notamos ainda que nenhuma operação de multiplicação é necessária nesta transformação (para o caso de $N=2^{M}$ ).

A adaptação de cada coeficiente $w_{i}^{\prime}(n)$ do equalizador, usando o algoritmo LMS multi-split (LMS-MS), pode ser descrita como [7]:

$$
w_{i}^{\prime}(n)=w_{i}^{\prime}(n-1)+\frac{\mu_{M S}}{r_{i}(n)} x_{i}^{\prime}(n) e(n),
$$

onde $i=0,1, \ldots, N-1, \mu_{M S}$ é o passo de adaptação,

$$
e(n)=d(n)-y(n)
$$

$$
y(n)=\sum_{i=0}^{N-1} x_{i}^{\prime}(n) w_{i}^{\prime}(n)
$$

e $r_{i}(n)$ é uma estimativa da variância de $x_{i}^{\prime}(n)$. Esta estimativa pode ser calculada por:

$$
r_{i}(n)=\frac{\sum_{j=1}^{n} \gamma^{n-j}\left|x_{i}^{\prime}(j)\right|^{2}}{\sum_{j=1}^{n} \gamma^{n-j}}=\frac{p_{i}(n)}{q_{i}(n)},
$$

onde $p_{i}(n)$ e $q_{i}(n)$ são calculados recursivamente por:

$$
\begin{gathered}
p_{i}(n)=\left|x_{i}^{\prime}(n)\right|^{2}+\gamma p_{i}(n-1), \\
q_{i}(n)=1+\gamma q_{i}(n-1),
\end{gathered}
$$

e $\gamma(0 \ll \gamma \leq 1)$ é um fator de esquecimento. O caso $\gamma=1$ é para um ambiente estacionário. Vale a pena observar que o cálculo de $r_{i}(n)$ feito aqui difere daquele apresentado em [7] e é computacionalmente mais estável.

\section{Processamento Largamente Linear: Conceitos FUNDAMENTAIS}

Seja uma seqüência $x(n)$ complexa, discreta, aleatória, com média nula. Usualmente, as estatísticas de segunda ordem de $x(n)$ são descritas pela função de autocovariância (FAC), que é definida por:

$$
\mu\left(n_{1}, n_{2}\right)=E\left\{x\left(n_{1}\right) x^{*}\left(n_{2}\right)\right\},
$$

onde (.)* é a operação de conjugação complexa. Entretanto, tem sido observado que a FAC não é inteiramente suficiente para descrever as estatísticas de segunda ordem em alguns casos [6, 11]. Logo, é necessário introduzir uma outra função chamada de pseudo-autocorrelação (FPAC), definida por:

$$
\rho\left(n_{1}, n_{2}\right)=E\left\{x\left(n_{1}\right) x\left(n_{2}\right)\right\}
$$

Então, essas duas funções, $\mu\left(n_{1}, n_{2}\right)$ e $\rho\left(n_{1}, n_{2}\right)$, são necessárias para uma descrição completa das estatísticas de segunda ordem de $x(n)$. Quando $\rho\left(n_{1}, n_{2}\right)$ é igual a zero, a FPAC pode ser omitida e o processo é conhecido como próprio. Por outro lado, se o processo apresenta uma FPAC não nula, então este processo é dito ser impróprio. Um processo impróprio é estacionário no sentido amplo (widesense stationary - WSS) se, e somente se, $E\{x(n)\}=m_{x}$ é uma constante e tanto a FAC quanto a FPAC são independentes do tempo discreto $n$ [11].

Deste modo, o processamento largamente linear faz uso de uma observação complexa imprópria $x(n)$, isto é, usa tanto $x(n)$ quanto o seu conjugado complexo $x^{*}(n)$. 


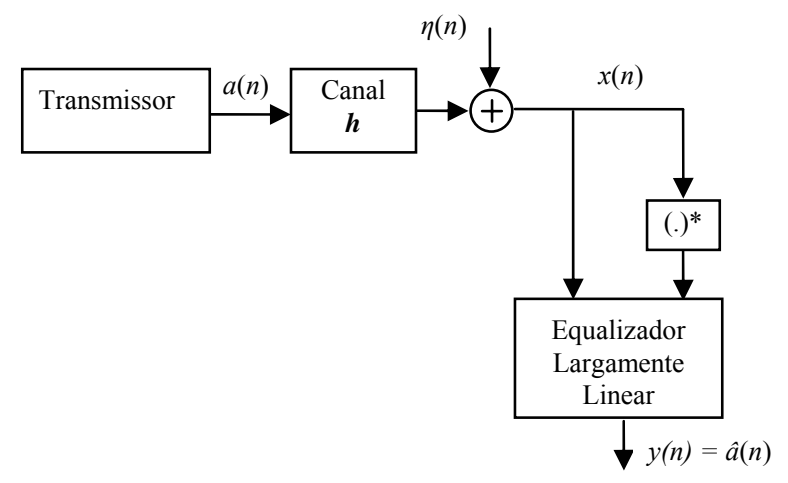

Fig. 3. Diagrama de blocos de um sistema de comunicação digital usando processamento largamente linear.

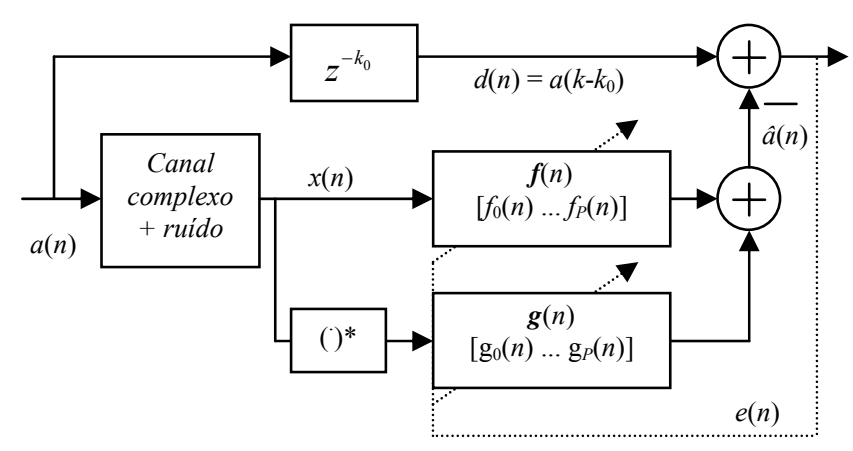

Fig. 4. Equalizador largamente linear treinado.

\section{EQUALIZAÇÃo LARGAMENTE LINEAR}

\section{A. Modelo do Sistema}

Neste artigo, consideramos o sistema de comunicação digital como descrito pela Fig. 3, onde $a(n), \eta(n), x(n)$ e $y(n)$ representam o sinal transmitido (com os símbolos pertencentes a uma constelação real e com média nula), o ruído (considerado complexo, com igual variância nas partes real e imaginária, aditivo, gaussiano, branco e independente de $a(n))$, o sinal recebido e a saída do equalizador, respectivamente.

O canal de comunicação digital, que pode ser representado por um filtro FIR (finite impulse response) de coeficientes complexos e comprimento $L$, apresenta os efeitos combinados do filtro de transmissão, do canal contínuo no tempo, filtro de recepção e da amostragem. Consideramos ainda que o transmissor gera uma seqüência de símbolos independentes identicamente distribuídos (i.i.d) e de variância $\sigma_{a}^{2}$. Já o ruído apresenta uma variância $\sigma_{\eta}^{2}$ e é considerado próprio.

O sinal recebido, após uma amostragem à taxa de símbolo e perfeita sincronização de portadora, é dado por:

$$
x(n)=\sum_{m=0}^{L} h(m) a(n-m)+\eta(n),
$$

onde $h(m)$ indica a resposta ao impulso do canal. Note que $x(n)$ é um processo impróprio e que o processamento largamente linear pode ser aplicado vantajosamente.

\section{B. Equalizador Largamente Linear}

O equalizador largamente linear utiliza além de $x(n)$ seu conjugado complexo $x *(n)$, como mostra a Fig. 4. De acordo com esta figura, o sinal de erro largamente linear pode ser expresso por

$$
\begin{aligned}
e_{L L}(n) & =d(n)-\sum_{m=0}^{P} f_{m}^{*}(n) x(n-m)-\sum_{m=0}^{P} g_{m}^{*}(n) x^{*}(n-m) \\
& =d(n)-\boldsymbol{f}^{H}(n) \boldsymbol{x}(n)-\boldsymbol{g}^{H}(n) \boldsymbol{x}^{*}(n),
\end{aligned}
$$

onde $d(n)=a\left(n-k_{0}\right)$ é o sinal de treinamento, $k_{0} \geq 0$ é um atraso que deve ser criteriosamente escolhido [12], $\boldsymbol{x}(n)=\left[\begin{array}{llll}x(n) & \ldots & x(n-M)\end{array}\right]^{T}, x(n)=\boldsymbol{h}^{T} \boldsymbol{a}(n)+\eta(n)$, com $\boldsymbol{h}=\left[\begin{array}{llll}h_{0} & h_{1} & \ldots & h_{L}\end{array}\right]^{T}, \quad \boldsymbol{f}(n)=\left[f_{0}(n) f_{1}(n) \ldots f_{P}(n)\right]^{T}$, $\boldsymbol{g}(n)=\left[g_{0}(n) \ldots g_{P}(n)\right]^{T}$ e $\boldsymbol{a}(n)=[a(n) \ldots a(n-L)]^{T}$.

A partir de (10) e usando as regras de derivação complexa [13] com o objetivo de minimizar a função custo $J=E\left\{\left|e_{L L}(n)\right|^{2}\right\}$, é fácil estabelecer o algoritmo LMS largamente linear como sendo expresso por $[3,8]$ :

$$
\begin{gathered}
\hat{a}(n)=\boldsymbol{f}^{H}(n) \boldsymbol{x}(n)+\boldsymbol{g}^{H}(n) \boldsymbol{x}^{*}(n) \\
e(n)=a\left(n-k_{0}\right)-\hat{a}(n) \\
\boldsymbol{f}(n+1)=\boldsymbol{f}(n)+\mu_{L L} e_{L L}^{*}(n) \boldsymbol{x}(n) \\
\boldsymbol{g}(n+1)=\boldsymbol{f}^{*}(n+1)
\end{gathered}
$$

onde $\mu_{L L}$ é o passo (fixo) de adaptação do algoritmo. Notamos que, para esta aplicação, o filtro $\boldsymbol{g}$ é uma versão conjugada complexa do filtro $\boldsymbol{f}$. Portanto, a complexidade computacional do algoritmo LMS-LL pode ser até menor que a sua versão estritamente linear, já que, em geral a ordem do equalizador precisa ser apenas igual a ordem do canal, ou seja, $P \geq L[1,3,14,15]$.

$\mathrm{O}$ algoritmo LMS-LL descrito em (13) pode ter o seu passo de adaptação $\mu_{L L}$ variável com o objetivo de acelerar a convergência do algoritmo [4, 8]. Talvez a técnica mais simples para tornar o passo variável seja normalizar o vetor $\boldsymbol{x}(n)$ :

$$
\mu_{L L}(n)=\mu_{N} \frac{\boldsymbol{x}(n)}{\varepsilon+\|\boldsymbol{x}(n)\|^{2}}
$$

onde $\varepsilon>0$ é um valor pequeno e $0<\mu_{N}<1$. Incluindo esta modificação em (13), teremos o algoritmo LMS-LL-N (LMS-LL normalizado). Também poderíamos usar outras técnicas de passo variável como, por exemplo, a indicada em [16], mas isto foge ao objetivo deste artigo.

Na próxima seção aplicaremos o procedimento multi-split ao equalizador largamente linear descrito e iremos comparar o desempenho deste novo esquema com os algoritmos LMS-LL e LMS-LL-N através de simulações. A Tabela I sumariza o algoritmo LMS-LL-MS. 
TABELA I: Algoritmo LMS-LL-MS

1) Transformação Linear: $\boldsymbol{x}^{\prime}(n)=\mathrm{H}_{N} \boldsymbol{x}$

2) Atualização:

$$
\begin{gathered}
y(n)=\boldsymbol{f}{ }^{, \mathrm{H}}(n) \boldsymbol{x}^{\prime}(n)+\boldsymbol{g}^{, \mathrm{H}}(n) \boldsymbol{x}^{*}(n) \\
e(n)=d(n)-y(n) \\
r_{i}(n)=p_{i}(n) / q_{i}(n) \quad(\text { ver equações } 6,7 \text { e } 8) \\
f^{\prime}{ }_{i}(n)=f^{\prime}{ }_{i}(n-1)+\mu_{M S} e(n) x^{*}{ }_{i}(n) / r_{i}(n) \\
\boldsymbol{g}^{\prime}(n)=\boldsymbol{f}{ }^{*}(n)
\end{gathered}
$$

\section{EqualizaÇ̃̃o LARGamente Linear Multi-Split e RESULtAdos De SimulaÇão}

A aplicação da operação multi-split no equalizador largamente linear é similar ao caso linear, bastando para isso aplicar a matriz de Hadamard nas partes reais e imaginárias dos vetores $\boldsymbol{x}(n)$ e $\boldsymbol{x}^{*}(n)$.

Para as simulações de Monte Carlo a seguir, iremos considerar que o sinal transmitido pertence a uma constelação 4-PAM, que o canal é complexo, discreto, de ordem $L$, e que a relação sinal-ruído (SNR - signal-to-noise ratio), definida por

$$
S N R=10 \log _{10}\left(\left(\sigma_{\eta}^{2}+\sigma_{a}^{2} \sum_{m=0}^{L}|h(m)|^{2}\right) / \sigma_{\eta}^{2}\right),
$$

é de $30 \mathrm{~dB}$. Todos os equalizadores considerados têm $P=8$ coeficientes. Em todas as simulações consideramos $\varepsilon=10^{-4}$ para o algoritmo LMS-LL-N e $\gamma=0,995$ para o algoritmo LMS-LL-MS. Escolhemos os passos de adaptação para que o erro quadrático médio $\left(E\left\{\left|e_{L L}(n)\right|^{2}\right\}, E\left\{\left|e_{L L-N}(n)\right|^{2}\right\}\right.$ e $\left.E\left\{\left|e_{L L-M S}(n)\right|^{2}\right\}\right)$ após a convergência dos algoritmos sejam aproximadamente os mesmos. Todas as curvas de aprendizado foram obtidas pela mediação de 400 realizações independentes.

\section{A. Simulação I}

Nesta primeira simulação, consideramos um canal cuja resposta ao impulso é $h_{l}(z)=0,601+(0,6309-0,2403 j) z^{-1}+$ $(0,355+0,024 j) z^{-2}+(-0,0348-0,047 j) z^{-3}+(0,156-0,1706 j) z^{-4}$. Este canal é de fase mista e apresenta um zero próximo ao círculo de raio unitário. A Fig. 5 mostra os zeros deste canal, bem como a sua resposta em freqüência, o sinal após passar pelo canal e o sinal equalizado. Podemos notar que o sinal equalizado é puramente real

A Fig. 6 apresenta as curvas de aprendizado para os algoritmos LMS-LL, LMS-LL-N (normalizado) e LMS-LLMS. Nesta simulação, escolhemos um atraso $k_{0}=3$. Notamos que os três algoritmos têm praticamente o mesmo erro quadrático médio final, mas o algoritmo LMS-LL-MS converge com um número menor de iterações. Os passos de adaptação usados foram: $\mu_{L L}=0,0045 ; \mu_{N}=0,22$ e $\mu_{L L-M S}=$ $1 / 32$.

\section{B. Simulação II}

Nesta segunda simulação, consideramos um canal cuja resposta ao impulso é dada por $h_{l}(z)=(0,3921+0,3921 j)+$ $(0,0392+0,745 j) z^{-1}+(0,051+0,2548 j) z^{-2}+(0,132+0,136 j) z^{-3}$
$+(0,1068+0,1129 j) z^{-4}+(0,0423+0,0819 j) z^{-5}$. Este canal também apresenta fase mista e um zero próximo ao círculo de raio unitário. A Fig. 7 mostra os zeros, a resposta em freqüência e o sinal após passar pelo canal.

A Fig. 8 apresenta as curvas de aprendizado para os algoritmos LMS-LL, LMS-LL-N e LMS-LL-MS. O atraso usado foi $k_{0}=2$. Novamente, observamos que os três algoritmos têm praticamente o mesmo erro quadrático médio após a convergência, mas o algoritmo LMS-LL-MS converge com um número menor de iterações. Os passos de adaptação usados foram: $\mu_{L L}=0,0050 ; \mu_{N}=0,25$ e $\mu_{L L-M S}=$ $1 / 32$.

\section{Simulação III}

Nesta última simulação, consideramos o canal definido por Porat e Friedlander [17], cuja resposta ao impulso é $h_{P}(z)=(0,485-0,0971 j)+(0,364+0,4368 j) z^{-1}+0,2427 z^{-2}+$ $(0,2912-0,3155 j) z^{-3}+(0,1941+0,3883 j) z^{-4}$. Este canal é de fase mista e apresenta dois zeros próximos ao círculo de raio unitário. A Fig. 9 mostra os zeros deste canal e a sua resposta em freqüência.

A Fig. 10 apresenta as curvas de aprendizado para os algoritmos LMS-LL, LMS-LL-N (normalizado) e LMS-LLMS. Escolhemos um atraso $k_{0}=4$. Notamos que, para este canal, o algoritmo LMS-LL-MS apresenta uma taxa de convergência apenas ligeiramente maior que a dos outros dois algoritmos. Os passos de adaptação usados foram: $\mu_{L L}$ $=0,0050 ; \mu_{N}=0,22$ e $\mu_{L L-M S}=1 / 32$.

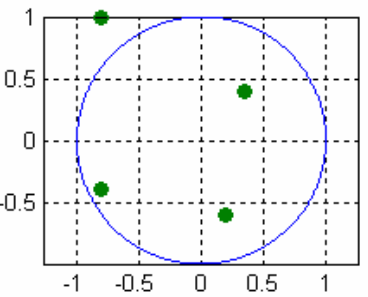

(a)

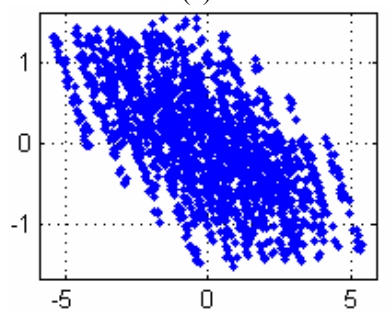

(c)

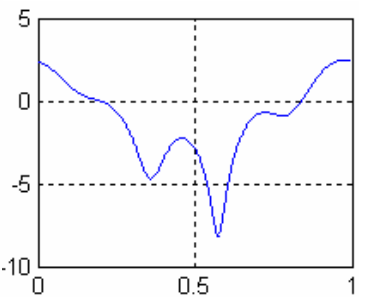

(b)

(d)

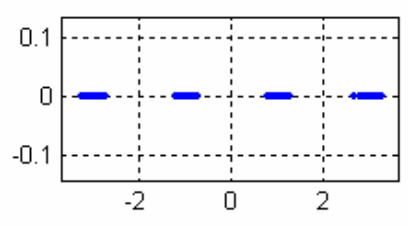

Fig. 5. (a) zeros do canal $\boldsymbol{h}_{1}$, (b) resposta em freqüência, (c) saída do canal, (d) constelação equalizada (igual para os três equalizadores).

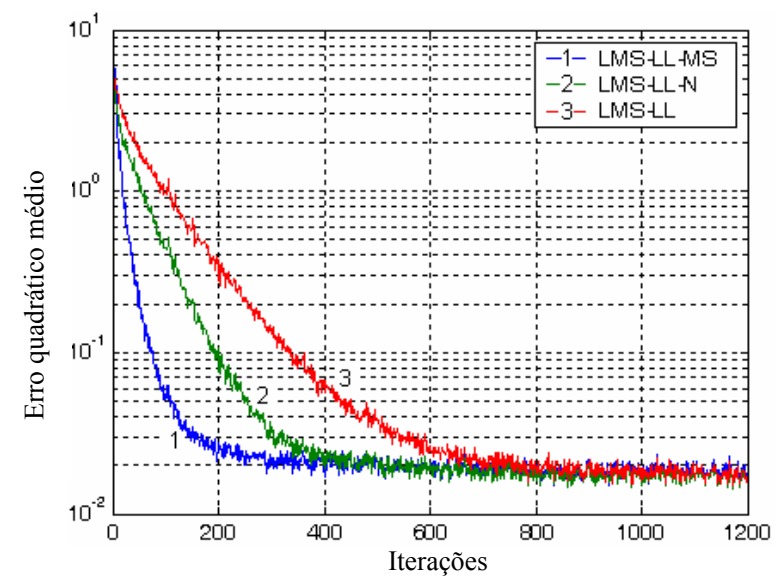

Fig. 6. Curvas de aprendizado para o canal $\boldsymbol{h}_{1}$. 


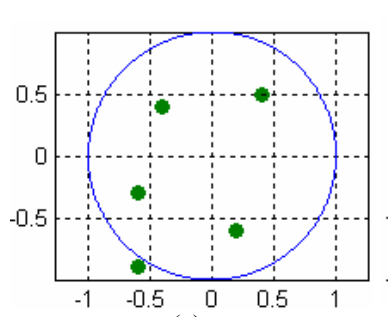

(a)

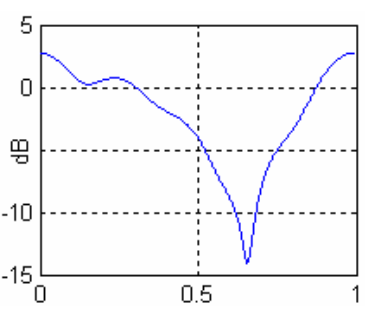

(b)

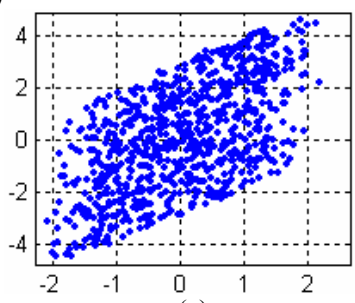

(c)

Fig. 7. (a) zeros do canal $\boldsymbol{h}_{2}$, (b) resposta em freqüência, (c) sinal após o canal.

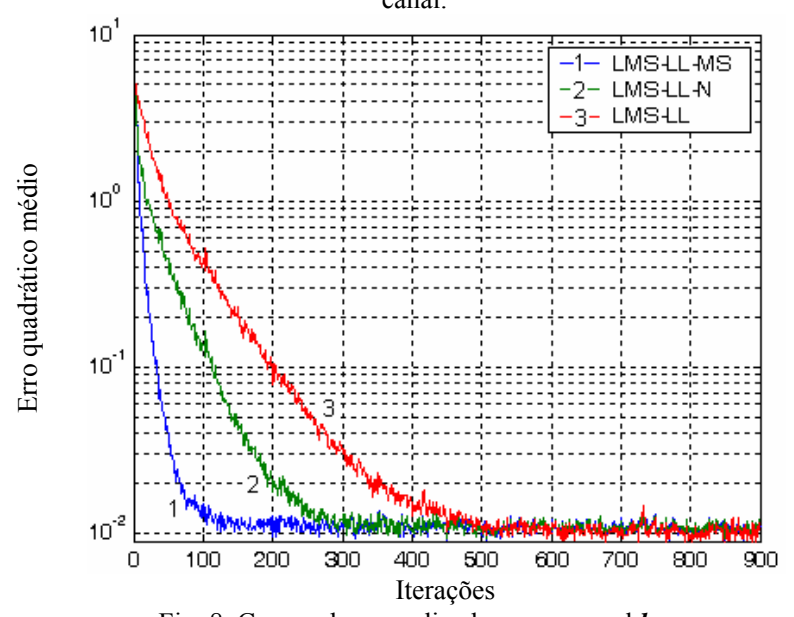

Fig. 8. Curvas de aprendizado para o canal $\boldsymbol{h}_{2}$.

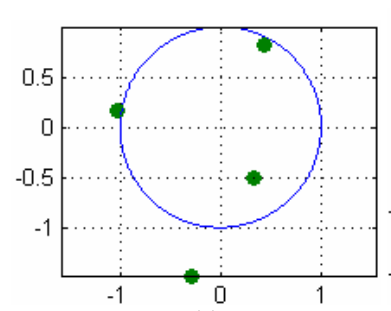

(a)

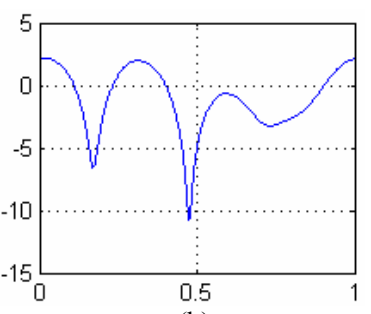

(b)
Fig. 9. (a) zeros do canal $\boldsymbol{h}_{P}$, (b) resposta em freqüência.

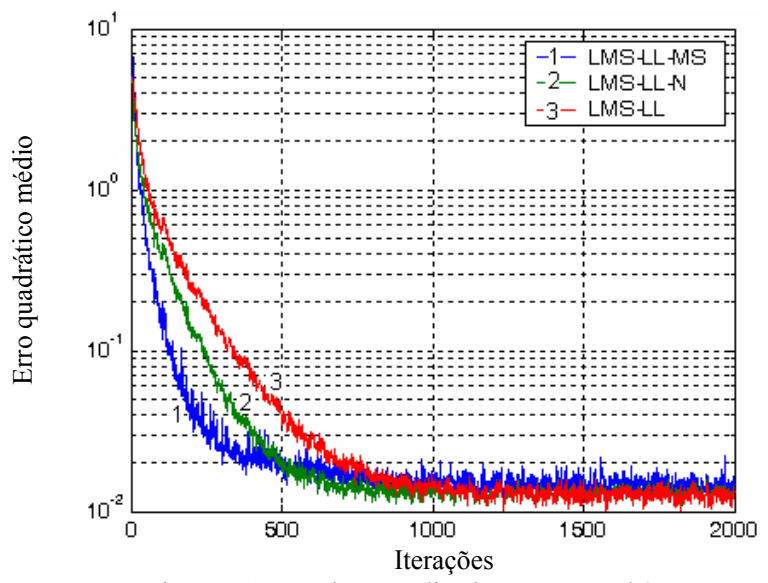

Fig. 10. Curvas de aprendizado para o canal $\boldsymbol{h}_{P}$.

\section{CONCLUSÃo}

Neste artigo, mostramos que a técnica multi-split pode ser aplicada ao algoritmo LMS largamente linear, da mesma forma que no caso linear. O desempenho do equalizador proposto foi avaliado para o caso de equalização adaptativa. Os resultados de simulação evidenciam que o algoritmo LMS-LL multi-split pode conseguir uma melhor taxa de convergência que a sua versão normalizada, para um desajuste final similar.

\section{REFERÊNCIAS}

[1] F. J. A. de Aquino, C. A. F. da Rocha e L. S. Resende, "Widely Linear Prediction Applied to Blind Equalization", Proceedings of International Workshop on Telecommunications (IWT) 2007, pp. 240-247, Feb. 2007.

[2] G. Gelli, L. Paura e A. R. P. Ragozini, "Blind widely linear multiuser detection," IEEE Commun. Lett., vol. 4, no. 6, pp. 187-189, Jun. 2000.

[3] W. H. Gerstacker, R. Schober e A. Lampe, "Receivers with widely linear processing for frequency-selective channels," IEEE Commun. Lett., vol. 7, no. 9, pp. 1512-1523, Sep. 2003.

[4] Amaro A. de Lima e Rodrigo C. de Lamare, "Adaptive Detection using Widely Linear Processing with Data Reusing for DS-CDMA Systems", VI International Telecommunications Symposium (ITS2006), Fortaleza-CE, Brazil, vol. 1, 167-172, Sep., 2006.

[5] R. Schober, W. H. Gerstacker, L. H.-J. Lampe, "Data-Aided and Blind Stochastic Gradient Algorithms for Widely Linear MMSE MAI Suppression for DS-CDMA", IEEE Trans. on Signal Processing, vol. 52, pp. 746-755, no. 3, Mar 2004.

[6] B. Picinbono, e P. Chevalier, "Widely linear estimation with complex data," IEEE Trans. Signal Processing, vol. 43, pp. 2030-2033, Aug. 1995.

[7] R.D. Souza, L.S. Resende, M.G. Bellanger, "Efficient Implementation of Multi-Split LMS Filtering with Complex Parameters", Proceedings of the 4th International Symposium on Image and Signal Processing and Analysis, pp. 419-423, 2005.

[8] F. J. A. de Aquino, C. A. F. da Rocha e L. S. Resende, "Accelerating the Convergence of the Widely Linear LMS Algorithm for Channel Equalization", VI International Telecommunications Symposium (ITS2006), Fortaleza-CE, Brazil, vol. 1, 57-61, Sep., 2006.

[9] K. F. Wan e P. C. Ching, "Multilevel split-path adaptive filtering and its unification with discrete Walsh transform application," IEEE Trans. Circuits Syst II, vol. 44, No.2, pp. 147-151, Feb. 1997.

[10] R. D. Souza, L. S. Resende, e M. G. Bellanger, "A justification for the improved performance of the multi-split LMS algorithm," in Proc. IEEE ICASSP'03, Hong Kong, pp. 89-92, Apr. 2003.

[11] F. D. Neeser e J. L. Massey, "Proper complex random processes with applications to information theory," IEEE Trans. Inform. Theory, vol. 39, pp. 1293-1302, July 1993.

[12] P.A. Voois, I. Lee, e J. M. Cioffi, "The effect of decision delay in finite-length decision feedback equalization", IEEE Transactions on Information Theory, Vol. 42, pp. 618-621, Mar. 1996.

[13] S. Haykin, "Adaptive Filter Theory", $4^{\text {th }}$. ed., Prentice Hall, New Jersey, 2002.

[14] C.B. Papadias e D.T.M. Slock, Fractionally spaced equalization of linear polyphase channels and related blind techniques based on multichannel linear prediction", IEEE Transactions on Signal Processing, Vol. 47, pp. 641-654, Mar. 1999.

[15] G.B. Giannakis, e S.D. Halford, "Blind fractionally spaced equalization of noisy FIR channels: direct and adaptive solutions", IEEE Trans. on Signal Processing, vol. 45, pp.:2277 - 2292, Sept. 1997.

[16] M.H. Costa e J.C. Bermudez, "A Robust Variable Step-Size Algorithm for LMS Adaptive Filters", IEEE Int. Conf. on Acoustics, Speech and Signal Processing, vol. 3, pp. 93-96, May 2006.

[17] B. Porat e B. Friedlander, "Blind Equalization of Digital Communication Channels Using High-Order Moments", IEEE Trans. on Signal Processing, vol. 39, no. 2, pp. 522-526, Feb. 1991 